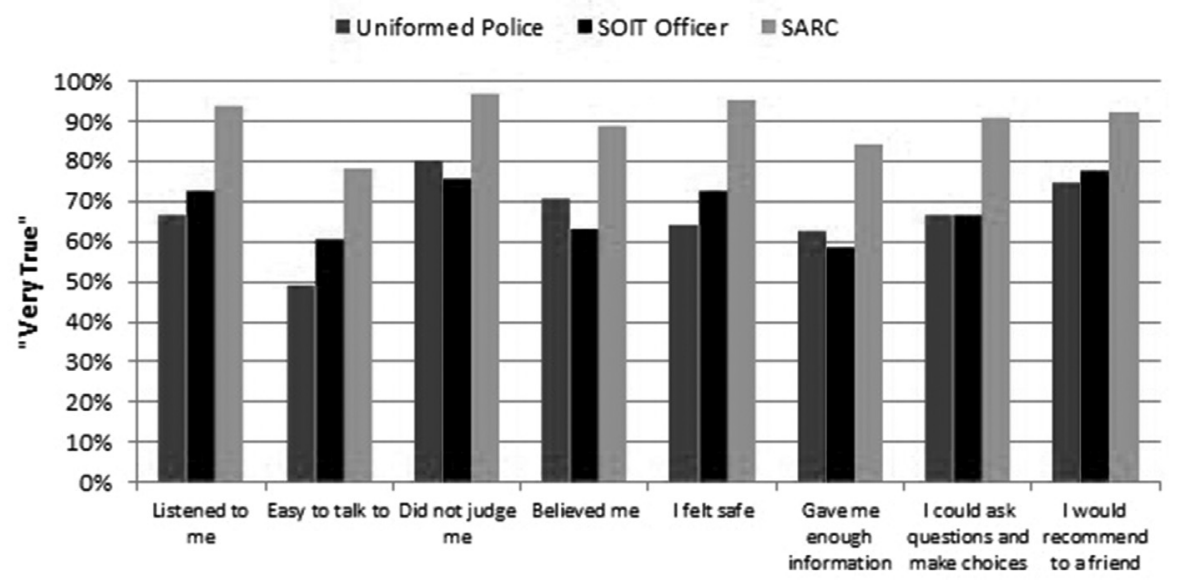

Abstract G147 Figure 1 Adolescent experiences of care following sexual assault

for Health Improvement Experience of Service Questionnaire (CHI-ESQ).

Results 30\% consented to take part. Data were analysed for the first 64 study participants, evaluating care received from uniformed police $(\mathrm{n}=51)$, specialist sexual offences investigative techniques (SOIT) officers $(n=58)$ and clinical staff at the SARCs (doctors and crisis workers, $\mathrm{n}=64$ ). $97 \%$ were police referrals and $3 \%$, self-referrals. $67 \%$ had experienced vaginal rape, $32 \%$, oral rape and $12 \%$, anal rape. $90 \%$ underwent a forensic medical examination.

Commonly held fears before accessing care were that they would be judged, disbelieved, 'blamed' or 'called a liar'. Many participants were apprehensive that the forensic examination would be painful, embarrassing or reveal health problems such as infections.

As shown in Figure 1, care given by clinical staff at SARCs was consistently rated higher than police care. Only $49 \%$ found uniformed police easy to talk to and only $64 \%$ felt safe in their care. Only $63 \%$ felt believed by their SOIT officer and only $59 \%$ felt they had been given enough information (Figure 1).

Conclusions Many participants described positive experiences of systems of care for victims of sexual assault. However, variable experiences of care between services highlight the need for improvement in specific areas. Anxieties about being blamed or judged may prevent adolescents accessing services following sexual assault.

\section{G148 THE RELATIONSHIP BETWEEN PUBERTAL STATUS AND NEURAL ACTIVITY DURING RISKY DECISION-MAKING IN MALE ADOLESCENTS USING FMRI}

\footnotetext{
${ }^{1,2} \mathrm{AL}$ Goddings, ${ }^{1,2} \mathrm{~L}$ Menzies, ${ }^{3}$ I Dumontheil, ${ }^{2} \mathrm{E}$ Garrett, ${ }^{2} \mathrm{~S} J$ Blakemore, ${ }^{1} \mathrm{RM}$ Viner. ${ }^{1}$ Institute of Child Health, University College London, London, UK; ${ }^{2}$ Institute of Cognitive Neuroscience, University College London, London, UK; ${ }^{3}$ Department of Psychological Sciences, Birkbeck College, University of London, London, UK
}

\subsection{6/archdischild-2015-308599.145}

During adolescence, risk-taking emerges as an important behaviour. One prominent theory proposes that this increased risk-taking results from a dissociation in the maturational timing of the brain regions involved in reward processing and cognitive control. It is hypothesised that the regions involved in reward processing, particularly the limbic system, mature relatively early in adolescence, in tandem with pubertal maturation. In contrast, the cognitive control regions of the brain, found principally within the prefrontal cortex, are proposed to undergo protracted development throughout adolescence. The goal of this functional Magnetic Resonance Imaging (fMRI) study was to explore how developmental changes in brain function when performing a risk-taking task were related to puberty, independently of chronological age.

Fifty male participants aged 12-14 years underwent fMRI scanning whilst performing a risk-taking task (the BART task). Indicators of pubertal development were collected, including self-reported pubertal status using Tanner stage diagrams and salivary hormone levels for testosterone, oestradiol and dehydroepiandrosterone (DHEA). Participants were grouped by pubertal status into early-mid puberty ( $\leq$ Tanner Stage 3 in pubic hair and gonadal development; $\mathrm{n}=23$ ) and late-post puberty ( $\geq$ Tanner Stage 4 in either pubic hair or gonadal development; $n$ $=26)$.

General linear modelling was used to investigate whether there were differences in neural activity within the reward processing and cognitive control brain networks during the risk-taking task with pubertal development, as measured by Tanner stage puberty groups and hormonal levels. Oestradiol levels were positively correlated with neural activity in the medial prefrontal cortex and orbitofrontal cortex when deciding not to make a risky choice. Testosterone levels and puberty grouping (early-mid vs. late-post) were correlated with prefrontal cortex activation during the processing of outcomes of a risky decision.

This study highlights the complexity of the decision-making process, and the extensive network of brain regions involved. It suggests that various aspects of the process of deciding to make a risky decision are related to distinct elements of pubertal development.

\section{G149 THE MANAGEMENT OF EATING DISORDERS ON CHILDREN'S WARDS: TRIALS, TRIBULATIONS AND TRAINING}

${ }^{1}$ A Yeadon, ${ }^{2}$ Mannion. ${ }^{1}$ School of Paediatrics, Health Education Yorkshire and the Humber, UK; ${ }^{2}$ Children's Ward, York Teaching Hospital NHS Foundation Trust, York, UK

\subsection{6/archdischild-2015-308599.146}

Aims This series of studies considers the challenges faced when caring for young people with eating disorders on children's wards, suggesting approaches to improving quality of care. 